Conclusion Our data show for the first time that high-frequency $(10 \mathrm{~Hz})$ cerebellar stimulation can produce long-lasting increases in human pharyngeal motor cortex excitability, with larger and longer-lasting effects of the intervention primarily seen in the contralateral projection. Hence $10 \mathrm{~Hz}$ cerebellar rTMS may play a therapeutic role in the treatment of dysphagia after hemispheric stroke.

Disclosure of Interest None Declared

\section{OC-037 DOES LEARNING OF ILLNESS BEHAVIOUR IN CHILDHOOD INFLUENCE THE DEVELOPMENT OF IRRITABLE BOWEL SYNDROME?}

doi:10.1136/gutjnl-2013-304907.037

1,"B T Theron, 'P Rastall, 'N Trudgill. 'Gastroenterology, Sandwell Hospital, West Bromwich, UK

Introduction We have previously shown that a twin with IBS was more likely to have a mother with IBS than a co-twin with IBS. This may be because learned illness behaviour in childhood is as important as genetic factors in the aetiology of IBS. We have examined the association of learned illness behaviour with IBS in our twin cohort.

Methods 6552 unselected twins were asked to complete a validated questionnaire including the social learning of illness scale. IBS was categorised by Rome III criteria into 3 groups: extended criteria IBS, limited criteria IBS (symptoms more than once a week) and a medical IBS diagnosis.

Results 4128 subjects replied (63\%). 3349 were evaluable (91\% female, median age 62 (IOR 53-69)). Univariate analysis revealed that only a medical diagnosis of IBS was associated with an increasing score on the social learning of illness scale (Table 1). Other factors associated with all three sub-types of IBS were increasing psychosomatic score, anxiety, female gender, younger age and having a parent with IBS. Social learning of illness behaviour and all these factors were independently associated with a medical diagnosis of IBS. Modelling of illness behaviour (OR 1.06, 95\% CI 1.01-1.12, $p=0.04$ ) and reinforcement of the sick role $(1.1,95 \%$ CI 1.01-1.2, $p=0.04)$ were found to be significant predictors of a medical diagnosis of IBS. However, encouragement of the sick role was not associated with a medical diagnosis of IBS.

Conclusion A higher score for social learning of illness behaviour was associated with a medical diagnosis of IBS. This was independent of other risk factors for IBS. In our twin cohort, the aspects of learned illness behaviour which influenced the development of IBS were reinforcement of the sick role and modelling of illness behaviour. Social learning of illness behaviour did not appear to influence IBS as defined by Rome III criteria when a medical diagnosis was not made, suggesting that it drives health care seeking behaviour rather than necessarily being an aetiological factor for IBS.

Disclosure of Interest None Declared

\section{Pancreatic free papers}

\section{OC-038 SPECIFIC MICRORNA MARKERS ARE IDENTIFIED IN BILE IN PANCREATIC DUCTAL ADENOCARCINOMA}

doi:10.1136/gutjnl-2013-304907.038

1.*A Zabron, ${ }^{2} \mathrm{~A}$ Frampton, ${ }^{3} \mathrm{~J}$ Krell, ${ }^{3} \mathrm{~J}$ Stebbing, ${ }^{3} \mathrm{~L}$ Castellano, ${ }^{1} \mathrm{~S}$ Khan, ${ }^{2} \mathrm{~L}$ Jiao. ${ }^{1}$ Hepatology and Gastroenterology Section, Department of Medicine; ${ }^{2}$ HPB Surgical Unit, Department of Surgery and Cancer; ${ }^{3}$ Division of Oncology, Department of Surgery and Cancer, Imperial College, London, UK

Introduction MicroRNAs (miRNAs) are short, non-coding RNAs with a key role in post-transcriptional regulation, and regulate multiple pathways of tumorogenesis. Expression profiling of malignant cell lines, fresh and formalin-fixed tumour have identified potential miRNA signatures for pancreatic ductal adenocarcinoma (PDAC), and RNA deep sequencing has demonstrated that miRNAs can be quantified from bile, in bilary tract cancers ${ }^{1}$. Here, we report the first study of biliary miRNAs in PDAC.

Methods 3 target miRNAs known to be overexpressed in PDAC were selected. Bile was collected at endoscopic retrograde cholangiopancreatography (ERCP) from patients with PDAC $(n=10)$ or gallstones ( $\mathrm{n}=8$; used as a benign control). Bile was prepared as described $^{1}$ and total RNA isolated using TRIzol (Invitrogen, Paisley, UK). Quantitative real-time reverse-transcription polymerase chain reaction (RT-qPCR) was performed using Taqman mature miRNA primers and probes (Applied Biosystems, Cheshire, UK). Expression of oncomiR-21, miR-155 and miR-106a was measured. Cycle passing threshold $(\mathrm{C} t)$ was recorded and normalised to RNU6B expression. Relative expression was calculated as $2^{\mathrm{Ct}}$ miRnA-Ct_RNU6B. PCR reactions were carried out in duplicate.

Results miR-106a and oncomiR-21 were both highly expressed in the bile of patients with PDAC, compared to those with gallstones. miR-155 was not significantly upregulated in PDAC bile. Using miR-106a (cut-off level > 8.02), we discriminated PDAC from benign disease with a sensitivity and specificity of $80 \%$ [95\% CI 44-98] and 100\% [95\% CI 63-100], respectively with an area under the curve (AUC) value of 0.88 . OncomiR-21 was also upregulated in bile, but was not as reliable in discriminating diagnosis.

Conclusion This study demonstrates that bile miRNAs are wellsuited analytes for the development of sensitive and specific tests in PDAC. Although tumour breach of the biliary epithelium was not always suspected in our cohort from clinical investigations, malignancy-specific miRNAs were found intraluminally. Of the 3 miRNAs tested, ROC analysis identified miR-106a as the best potential PDAC biomarker, with reliable diagnostic specificity and sensitivity. However, further study may also identify miRNAs that also discriminate prognoses and response to therapy, guiding individualised treatment. Disclosure of Interest None Declared

\section{REFERENCE}

1. Shigehara K, Yokomuro S, Ishibashi 0 , et al. Real-time PCR-based analysis of the human bile microRNAome identifies miR- 9 as a potential diagnostic biomarker for biliary tract cancer. PloS one. 2011; 6(8):e23584.

Abstract OC-037 Table 1 Univariate analysis of factors associated with IBS

\begin{tabular}{llll}
\hline VARIABLE & ROMEIII EXTENDED CRITERA IBS & ROME III LIMITED CRITERIA IBS & MEDICAL DIAGNOSIS IBS \\
\hline $\begin{array}{l}\text { Social Learning of IIIness Behaviour } \\
\text { Scale }\end{array}$ & $0.89(0.66-1.21, \mathrm{p}=0.47)$ & $1.00(0.99-1.01, \mathrm{p}=0.5)$ & $1.15(1.13-1.17, \mathrm{p}<0.01)$ \\
Psychosomatic score & $1.19(1.17-1.21, \mathrm{p}<0.001)$ & $1.21(1.19-1.24, \mathrm{p}<0.001)$ & $1.15(1.13-1.17, \mathrm{p}<0.001)$ \\
Anxiety & $1.75(1.47-2.09, \mathrm{p}<0.001)$ & $1.54(1.19-1.98, \mathrm{p}=0.001)$ & $1.84(1.40-2.42, \mathrm{p}<0.001)$ \\
Female gender & $2.16(1.54-3.03, \mathrm{p}<0.001)$ & $2.94(1.48-5.85, \mathrm{p}=0.002)$ & $2.19(1.18-4.05, \mathrm{p}<0.001)$ \\
Age & $0.99(0.98-0.99, \mathrm{p}=0.001)$ & $1.03(1.03-1.04, \mathrm{p}<0.001)$ & $1.11(1.10-1.12, \mathrm{p}<0.001)$ \\
Parent IBS & $2.48(2.00-3.08, \mathrm{p}<0.001)$ & $1.81(1.35-2.43, \mathrm{p}<0.001)$ & $2.72(2.15-3,44, \mathrm{p}<0.001)$ \\
\hline
\end{tabular}

